\title{
SUR L'INTÉGRALE FINIE D'UNE FONCTION ENTIERE
}

$$
\text { PAR }
$$

\section{A. HURWITZ}

$$
\text { a Z Z̈RICH. }
$$

D'après AbEx nous entendons par l'intégrale finie d'une fonction $G(z)$ la solution la plus générale de l'équation

$$
F(z+\mathfrak{r})-F(z)=G(z) .
$$

$F(z)$ étant une solution particulière de cette équation, la solution la plus générale sera évidemment $F(z)+\varphi(z)$, où $\varphi(z)$ désigne une fonction arbitraire qui admet la période $I$.

Dans ce qui suit nous nous occuperons de l'équation ( $I$ ) en supposant que la fonction donnée $G(z)$ est une fonction entière, c'est à dire que $G(z)$ peut être représentée par une série entière

$$
G(z)=a_{0}+a_{1} z+a_{2} z^{2}+\ldots
$$

convergente dans tout le plan. Le même cas fait l'objet principal d'un mémoire de M. C. Guichakd. ${ }^{1}$ En s'aidant des intégrales à coupures de M. Hermite l'éminent géomètre prouve que, $G(z)$ étant une fonction entière, il existe toujours une solution $F(z)$ de l'équation (I) qui est ellemême une fonction entière. J'arrive au même résultat en suivant une voie toute différente. On verra que le même ordre d'idées que celui qui fait la base du théorème de M. MittaG-Leffier m'amène au but.

1 Sur la résolution de l'équation aux différences finies $G(x+1)-G(x)=H(x)$. Annales scientifiques de l'Ecole Normale Supérieure. $3^{\text {me }}$ série, t. 4. (I 887.$)$ Acta mathematica. 20. Imprimê le 26 janvier 1897. 
Soit $\varphi_{n}(z)$ la fonction de Bewoulul, c'est à dire la fonction rationelle entière de degré $u+1$ qui s'annule pour $z=0$ et qui satisfait à l'équation

$$
\varphi_{n}(z+1)-\varphi_{n}(z)=z^{n} .
$$

Il est évident que la série

$$
F(z)=a_{0} \varphi_{0}(z)+a_{1} \varphi_{1}(z)+a_{2} \varphi_{2}(z)+\ldots
$$

satisfait formellement à l'équation (I). Or, d'après un théorème fondamental de M. Weierstrass, la série (4) définit une fonction entière $F(z)$, si elle converge uniformément dans toute région finie du plan. Ainsi, dans ce cas, nous n'avons rien à démontrer.

Supposons maintenant que la série (4) ne satisfusse pas aux conditions du théorème de $\mathrm{M}$. Weierstrass. Considérons alors au lieu de la série (4) la série suivante

(4') $\quad F(z)=a_{0}\left(\varphi_{0}(z)-\pi_{0}(z)\right)+a_{1}\left(\varphi_{1}(z)-\pi_{1}(z)\right)+a_{2}\left(\varphi_{2}(z)-\pi_{2}(z)\right)+\ldots$, $\pi_{0}(z), \pi_{1}(z), \pi_{2}(z), \ldots$ désignant des fonctions rationelles entières de $e^{2 \pi i z}$ et $e^{-2 \pi i z}$. Cette nouvelle série, coinme l'ancienne, satisfait formellement à l'équation ( $\mathrm{I}$ ). Nous ferons voir par les considérations qui suivent que l'on peut toujours disposer des fonctions $\pi_{0}(z), \pi_{1}(z), \pi_{2}(z), \ldots$ de façon que la série $\left(4^{\prime}\right)$ soit uniformément convergente dans toute région finie du plan et ainsi, le théorème de M. Guichard sera démontré. Il suffit comme nous verrons, de prendre pour $\pi_{n}(z)$ un certain nombre de termes de la série de Fourier représentant dans l'intervalle réel $0<z<$ I la fonction $\varphi_{n}(z)$.

Remarquons d'abord que la fonction $\varphi_{n}(z)$ est le coefficient de $\frac{\zeta^{n}}{\Gamma_{n}}$ dans le développement suivant les puissances entières de $\zeta$ de la fonction

$$
f(z, \zeta)=\frac{e^{z \zeta}-1}{e^{\tau}-1}
$$

C'est un fait connu, qui résulte d'ailleurs immédiatement des équations

$$
f(z+\mathrm{I}, \zeta)-f(z, \zeta)=e^{2 \zeta}, \quad f(0, \zeta)=0 .
$$


Ainsi nous avons

$$
f(z, \zeta)=\varphi_{0}(z)+\varphi_{1}(z) \frac{\zeta}{\underline{I}}+\varphi_{2}(z) \frac{\zeta^{2}}{\underline{2}}+\cdots
$$

pour toute valeur finie de $z$ et toute valeur de $\zeta$ satisfaisant à la condition $|\zeta|<2 \pi$. Par conséquent nous avons aussi

$$
\varphi_{n}(z)=\frac{\mid n}{2 \pi i} \int f(z, \zeta) \frac{d \zeta}{\zeta^{n+1}},
$$

l'intégrale étant prise dans le sens direct autour du point $\xi=0$.

Maintenant soit $r$ un nombre entier nul ou positif et $C_{r}$ une courbe fermée contenant à son intérieur les points

$$
0, \pm 2 \pi i, \pm 4 \pi i, \ldots, \pm 2 r \pi i
$$

tandis que les points $\pm 2 k \pi i(k>r)$ sont à son extérieur. Considérons l'intégrale

$$
\phi_{n, r}(z)=\frac{\mid n}{2 \pi i} \int_{C_{r}} f(z, \zeta) \frac{d \xi}{\zeta^{n+1}}
$$

qui se réduit à l'intégrale (7) pour $r=0$. En appliquant le théorème de Cauchy nous trouvons

$$
\psi_{n, r}(z)=\varphi_{n}(z)-\pi_{n, r}(z),
$$

où nous avons posé

$$
\pi_{n, r}(z)=\mid \underline{n} \sum \frac{1-e^{2 k \pi i z}}{(2 k \pi i)^{n+1}} . \quad(k= \pm 1, \pm 2, \ldots, \pm r)
$$

Soit $R$ une région finie quelconque du plan des $z$. On a le théorème suivant qui est fondamental pour notre objet:

Pour chaque valeur de $z$ qui appartient ì la région $R$ on $a$

$$
\left|\psi_{n, r}(z)\right|<\frac{16 \rho}{\pi} \cdot \frac{\mid n}{(2 r+1)^{n}} \frac{\rho^{2 r}}{\pi^{n}},
$$

$\rho$ désignant une quantité positive ne dépendant que de la région $R$ et nullement de $n$ et de $r$. 
Pour le démontrer, choisissons le contour d'intégration $C_{r}$ de l'intégrale (8) de la manière suivante. Posons $;=x+i y$ et, pour abréger,

$$
\lambda=(2 r+1) \pi \text {. }
$$

Le contour $C_{r}$ sera formé par les lignes

$$
x=\lambda, \quad y=\lambda, \quad x=-\lambda, \quad y=-\lambda .
$$

$\mathrm{Si} \zeta=x+i y$ parcourt le contour $C_{r}$, le point $\frac{\zeta}{\lambda}$ décrit le contour du carré dont les côtés sont

$$
x=\mathrm{I}, \quad y=\mathrm{I}, \quad x=-\mathrm{I}, \quad y=-\mathrm{I} .
$$

Ainsi nous aurons, en désignant par $U$ ce dernier contour,

$$
\psi_{n, r}(z)=\frac{\mid n}{\lambda^{n}} \cdot \frac{1}{2 \pi i} \int_{U} \frac{e^{\lambda z \zeta}-\mathrm{I}}{e^{\lambda \zeta}-1} \frac{d \zeta}{\zeta^{n+1}} .
$$

Déterminons maintenant la quantité positive $\rho$ supérieure à l'unité de manière que l'on ait

$$
\rho>\left|e^{z ; \pi}\right|
$$

pour toutes les valeurs de $z$ situées dans la région $R$ et pour celles de $\zeta$ qui appartiennent au contour $U$ d'intégration. Nous aurons évidemment

$$
\left|e^{\lambda z}-\mathrm{I}\right| \leqq\left|e^{s ; \pi}\right|^{2 r+1}+\mathrm{I}<2 \rho^{2 r+1} \text {. }
$$

Le long des côtés (13) du contour $U$ les valeurs absolues de $e^{\lambda \zeta}-\mathrm{t}$ sont respectivement supérieures ou égales à

$$
e^{\lambda}-1, \mathrm{I}+e^{-\lambda}, \mathrm{I}-e^{-\lambda}, \mathrm{I}+e^{-\lambda}
$$

Il en résulte qu'on aura constamment sur le chemin d'intégration

$$
\left|e^{\lambda \xi}-\mathbf{I}\right| \geqq \mathbf{I}-e^{-\lambda} \geqq \mathbf{I}-e^{-\pi}>\frac{\mathrm{I}}{2} \text {. }
$$

Enfin, le module de $\zeta$ étant supérieur à l'unité le long de $U$, nous trouvons

$$
\left|\psi_{n, r}(z)\right|<\frac{\mid n}{\lambda^{n}} \cdot \frac{\mathrm{I}}{2 \pi} 4 \rho^{2 r+1} \int_{U}|d \zeta|=\frac{\mathrm{I} 6 \rho}{\pi} \frac{\mid \underline{n}}{(2 r+\mathrm{I})^{n}} \frac{\rho^{2 r}}{\pi^{n}},
$$

ce qu'il fallait démontrer. 
Ceci posé, soit

$$
G(z)=a_{0}+a_{1} z+\ldots+a_{n} z^{n}+\ldots
$$

une fonction entière quelconque; formons la série

$$
\begin{gathered}
F(z)=a_{0}\left(\varphi_{0}(z)-\pi_{0,0}(z)\right)+a_{1}\left(\varphi_{1}(z)-\pi_{\mathrm{i}, 1}(z)\right)+\ldots \\
+a_{n}\left(\varphi_{n}(z)-\pi_{n, u}(z)\right)+\ldots,
\end{gathered}
$$

qui satisfait, comme nous l'avons déjà remarqué, formellement à l'équation

$$
F(z+\mathrm{I})-F(z)=G(z) .
$$

Je dis que la série (I5) converge absolument et uniformément dans toute région finie $R$. En effet, $\varphi_{n}(z)-\pi_{n, n}(z)$ étant égal à $\psi_{n, n}(z)$, le terme général de la série (I 5) est, en valeur absolue, inférieur à

$$
\frac{16 \rho}{\pi}\left|a_{n}\right| \frac{\mid n}{(2 n+1)^{n}}\left(\frac{\rho^{2}}{\pi}\right)^{n}
$$

pour une valeur quelconque de $z$ appartenant à la région $R$. Il suffira donc de faire voir que la série

$$
\sum_{0}^{\infty}\left|a_{n}\right| \frac{\mid n}{(2 n+1)^{n}} z^{n}
$$

converge pour $z=\frac{\rho^{2}}{\pi}$. Or cette série converge dans tout le plan. En effet, pour qu'une série entière soit toujours convergente il faut et il suffit que la racine $n^{\text {ième }}$ du coefficient $n^{\text {ième }}$ tende vers zéro avec $\frac{I}{n}$. Cela découle immédiatement du théoréme de M. Hadamand sur le rayon du cercle de convergence d'une série entière. Maintenant, $\sum_{0}^{\infty} a_{n} z^{n}$ étant d'après l'hypothèse toujours convergente, on a

$$
\lim _{n \rightarrow \infty} \sqrt[n]{a_{n}}=0
$$

De plus la formule de Stiruing

$$
\underline{n}=\sqrt{2 \pi} n^{n+\frac{1}{2}} e^{-n+\frac{\theta}{12 n}}, \quad(0<\theta<1)
$$


donne aisément

$$
\operatorname{Lim}_{n=\infty} \sqrt[n]{\frac{\mid n}{(2 n+1)^{n}}}=\frac{1}{2 e}
$$

Par conséquent la racine $n^{\text {tème }}$ de $\left|a_{n}\right| \frac{\mid n}{(2 n+\mathrm{I})^{n}}$ tend vers zéro avec $\frac{\mathrm{I}}{n}$; donc la série ( 16 ) est toujours convergente.

Ainsi la série (15) définit bien une fonction entière $F(z)$ satisfaisant à l'équation $F(z+\mathrm{I})-F(z)=G(z)$. Résumons ce résultat de la manière suivante:

Soit

$$
G(z)=\sum_{n=0}^{\infty} a_{n} z^{n}
$$

une fonction entière quelconque. Désignons par $\varphi_{n}(z)$ la fonction de Bernoulli et par $\pi_{n, r}(z)$ la fonction rationnelle entière de $e^{2 \pi i z}$ et $e^{-2 \pi i z}$ définie par l'équation

$$
\pi_{n, r}(z)=\ln \sum \frac{1-e^{2 k-i z}}{(2 k \pi i)^{n+1}}
$$

Alors, les indices $r$ étant choisis d'une manière convenable, la série

$$
F(z)=\sum_{n=0}^{\infty} a_{n}\left[\varphi_{n}(z)-\pi_{n, r}(z)\right]
$$

converge uniformément dans toute région finie du plan et représente par conséquent une fonction entière satisfaisant à l'équation

$$
F(z+\mathrm{I})-F(z)=E(z) \text {. }
$$

On peut, d'après l'analyse précédente, prendre $r=n$, quelle que soit la fonction $G(z)$. Dans quelles conditions est-il possible de prendre pour l'indice $r$ une valeur constante, ne variant pas avec l'indice $n$ ? C'est une question qui - comme la question analogue dans le théorème de M. Mittag-Leffler - se présente naturellement et que je vais aborder dans les lignes qui suivent.

Soit

$$
G(z)=\sum_{n=0}^{\infty} a_{n} z^{n}
$$


une fonction entière et posons

$$
\begin{gathered}
F(z)=a_{0}\left[\varphi_{0}(z)-\pi_{0, r}(z)\right]+a_{1}\left[\varphi_{1}(z)-\pi_{1, r}(z)\right]+\ldots \\
+a_{n}\left[\varphi_{n}(z)-\pi_{n, r}(z)\right]+\ldots
\end{gathered}
$$

Je dis que la condition nécessaire est suffisante pour que l'on puisse donner à l'indice $r$ une valeur telle que la série ( 17 ) converge uniformément dans toute région finie du plan est que le rayon du cercle de convergence de la série

$$
\sum_{n=0}^{\infty} \mid \underline{n} a_{n} z^{n}
$$

ne s'évanouisse pas.

En premier lieu je vais démontrer que c'est une condition nécessaire.

Supposons que la série ( 7 ) soit uniformément convergente dans une région contenant les valeurs réclles de $z$ satisfaisant à la condition $0 \leqq z \leq \mathrm{I}$ (ce qui a certainement lieu, si la série (I 7 ) est uniformément convergente dans toute région finie du plan). Alors la fonction $F(z)$ représentée par la série (17) est une fonction continue dans l'intervalle $0 \leqq z \leq 1$ et peut être développée d'après le théorème de Fourier suivant les sinus et cosinus des multiples de $2 \pi z$. Pour plus de simplicité introduisons la fonction exponentielle au lieu des fonctions trigonométriques et posons

$$
F(z)=\frac{\mathrm{I}}{2} \alpha_{0}+\left(\alpha_{1} e^{2 \pi i z}+\alpha_{-1} e^{-2 \pi i z}\right)+\ldots+\left(\alpha_{k} e^{2 k \pi i z}+\alpha_{-k} e^{-2 k \pi i z}\right)+\ldots
$$

où les coefficients $\alpha_{k}$ sont à déterminer d'après la formule

$$
a_{k}=\int_{0}^{1} F(z) e^{-2 k \pi i z} d z: \quad(k \approx 0, \pm 1, \pm 2, \ldots)
$$

Or la série ( I 7 ) étant uniformément convergente dans l'intervalle $0 \leqq z \leq 1$, nous obtenons l'intégrale (20) en intégrant terme à terme la série (I 7) après l'avoir multipliée par $e^{-2 k \pi i z}$. Supposons $|k|>0$; alors l'équation (8) nous donne:

$$
\int_{0}^{1}\left[\varphi_{n}(z)-\pi_{n, r}(z)\right] e^{-2 k \pi i z} d z=\frac{\mid n}{2 \pi i} \int_{C r} \frac{d \zeta}{\zeta^{n+1}(\zeta-2 k \pi i)}
$$


L'intégrale s'annule pour $|k| \leqq r$, puisque la fonction $\frac{\mathrm{I}}{\zeta^{n+1}(\zeta-2 k \pi i)}$, holomorphe en dehors de $C_{r}$, devient infiniment petite d'un ordre supérieur à 1 pour $\zeta=\infty$.

$\mathrm{Si}|k|>r$ la valeur de lintégrale est

$$
-\frac{\mid n}{(2 k \pi i)^{n+1}}
$$

la fonction $\frac{1}{\zeta^{n+1}(\zeta-2 h \pi i)}$ ayant alors en dehors de $C_{r}$ le pôle $\zeta=2 k \pi i$.

Ainsi nous aurons

$$
\alpha_{ \pm 1}=\alpha_{ \pm 2}=\ldots=\alpha_{ \pm r}=0
$$

et, pour $|k|>r$,

$$
a_{k}=-\sum_{n=0}^{\infty} \mid \underline{n} a_{n} \frac{1}{(2 k \pi i)^{n+1}} .
$$

Il en résulte que la série (18) est convergente pour $|z|<\frac{\mathrm{I}}{2 \pi(r+1)}$. De plus nous pouvons énoncer la proposition suivante:

Si la série

$$
\begin{gathered}
F(z)=a_{0}\left[\varphi_{0}(z)-\pi_{0, r}(z)\right]+a_{1}\left[\varphi_{1}(z)-\pi_{1, r}(z)\right]+\ldots \\
+a_{n}\left[\varphi_{n}(z)-\pi_{n, r}(z)\right]+\ldots
\end{gathered}
$$

est uniformément convergente dans une région contenant l'intervalle réel $0 \leqq z \leq 1$, la série

$$
g(z)=\sum_{n=0}^{\infty} \ln a_{n} \frac{1}{(2 \pi i z)^{n+1}}
$$

sera convergente pour $z= \pm(r+1)$ et par conséquent pour $|z|>r+1$. En outre le développement de Fourier pour $F(z)$ dans l'intervalle $\mathrm{O}<z<\mathrm{I}$ sera

$$
F(z)=\frac{1}{2} \alpha_{0}-\sum_{n=r+1}^{\infty}\left\{g(n) e^{2 n-i z}+g(-n) e^{m: n \pi i z}\right\} .
$$

La valeur de $\alpha_{0}$ peut ètre déterminée par l'intégrale (20), ou, ce qui est plus commode, de la manière suivante. La série de Fourier 
pour $z=0$ donne la valeur $\frac{1}{2}(F(0)+F(\mathrm{I}))$. Or cette valeur d'après (22) est égal à $\frac{1}{2} a_{0}$ puisque les fonctions $\varphi_{n}(z)$ et $\pi_{n, r}(z)$ sont, à l'exception de $\varphi_{0}(z)=z$, nulles pour $z=0$ et $z=\mathrm{I}$. Ainsi nous avons pour $\alpha_{0}$ l'équation

$$
\frac{\mathrm{I}}{2} \alpha_{0}=\frac{\mathrm{I}}{2} a_{0}+\sum_{n=r+1}^{\infty}\{g(n)+g(-n)\} .
$$

Maintenant supposons inversement, que,

$$
G(z)=\sum_{n=0}^{\infty} a_{n} z^{n}
$$

étant une fonction entière, la série (I 8) ait un rayon de convergence différent de zéro. Alors la série

$$
g(z)=\sum_{n=0}^{\infty} \ln _{-} a_{n} \frac{1}{(2 \pi i z)^{n+1}}
$$

converge pour $|z|>l, l$ étant un nombre positif fini. Désignons par $r+\mathrm{I}$ le premier nombre entier positif supérieur à $l$. Je dis que la série

$$
F(z)=\sum_{n=0}^{\infty} a_{n}\left[\varphi_{n}(z)-\pi_{n, r}(z)\right]
$$

est uniformément convergente dans toute région finie du plan.

Pour le démontrer appliquons la formule (8), que nous transformons

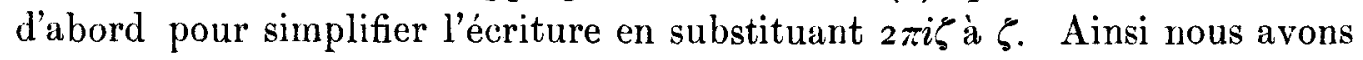

$$
\varphi_{n}(z)-\pi_{n, r}(z)=\int \frac{e^{2 \pi i z \zeta}-1}{e^{2 \pi i \zeta}-1} \frac{\mid n}{(2 \pi i \zeta)^{n+1}} d \zeta
$$

Nous prenons comme contour de l'intégration un cercle dont le centre est à l'origine et dont le rayon est compris entre $l$ et $r+1$. Cela posé, la somme des $n$ premiers termes de la série (27) sera égale à

$$
S_{n}(z)=\int \frac{e^{2 \pi i z_{\zeta}}-\mathrm{I}}{e^{2 \pi i \zeta}-\mathrm{I}} y_{n}(\zeta) d \zeta
$$

$g_{n}(z)$ désignant la somme des $n$ premiers terme de la série (26). Mais 
celle-ci étant uniformément convergente le longr du contour d'intégration, il est évident que pour $n=\infty S_{n}(z)$ tend uniformément dans toute région finie $\mathrm{du}$ plan des $z$, vers l'intégrale

$$
\int \frac{e^{1 \pi i z \zeta}-\mathrm{I}}{e^{2 \pi i \zeta}-\mathrm{I}} g(\zeta) d \zeta
$$

Ainsi nous obtenons la proposition suivante:

Supposons que,

$$
G(z)=a_{0}+a_{1} z+\ldots+a_{n} z^{n}+\ldots
$$

étant une fonction entière, la série

$$
g(z)=\left\|_{0} \frac{1}{2 \pi i z}+a_{1} \frac{1}{(2 \pi i z)^{2}}+\ldots+\right\|_{1} a_{n} \frac{1}{(2 \pi i z)^{n+1}}+\ldots
$$

soit convergente pour $|z|>l$. Soit de plus $r+1$ le premier nombre entier positif supérieur à $l$. Alors la série

$$
\begin{gathered}
F(z)=a_{0}\left[\varphi_{0}(z)-\pi_{0, r}(z)\right]+a_{1}\left[\varphi_{1}(z)-\pi_{1, r}(z)\right]+\ldots \\
+a_{n}\left[\varphi_{n}(z)-\pi_{n, r}(z)\right]+\ldots
\end{gathered}
$$

est uniformément convergente dans toute région finie du plan et représente par conséquent une fonction entière satisfaisant à l'équation $F(z+1)-F(z)=G(z)$. En outre on a

$$
F(z)=\int \frac{e^{2 \pi i z \zeta}-1}{e^{2 \pi i \zeta}-1} g(\zeta) d \zeta,
$$

l'intégrale étant prise le long d'un cercle dont le centre est à l'origine et dont le rayon est compris entre $l$ et $r+1$.

Le développement en série de Fourier de la fonction $F(z)$ est donné par les équations (24) et (25).

Ajoutons encore quelques remarques qui se rattachent aux théorèmes démontrés. La dérivée $F^{\prime}(z)$ d'une fonction $F(z)$ vérifiant l'équation $F(z+1)-F(z)=G(z)$ sera évidemment une solution de l'équation

$$
F^{\prime}(z+1)-F^{\prime}(z)=G^{\prime}(z) \text {. }
$$


Ainsi nous pouvons résoudre la dernière équation de deux manières différentes: soit directement en appliquant les résultats précédents, soit indirectement en résolvant l'équation $F(z+1)-F(z)=G(z)$ et prenant alors la dérivée de la solution trouvée. Je distinguerai les deux solutions de l'équation (33) ainsi obtenues comme première et deuxième solution. Elles ne peuvent différer que par une fonction entière ayant la période $\mathbf{I}$. Il s'agit de déterminer celle-ci. Pour plus de simplicité je me place dans le cas du dernier théorème. Ainsi je suppose que la fonction $F(z)$ soit représentée par l'intégrale (32). Alors la deuxième solution de l'équation (33) sera

$$
F^{\prime}(z)=\int \frac{e^{2 \pi i z \zeta}}{e^{2 \pi i \zeta}-1} 2 \pi i \zeta g(\zeta) d \zeta
$$

Pour avoir la première solution, que je nommerai $F_{1}(z)$, il faut former la fonction $g_{1}(z)$ qui dépend de

$$
G^{\prime}(z)=\sum_{n=0}^{\infty}(n+\mathrm{I}) a_{n+1} z^{n}
$$

de la même manière que $g(z)$ dépend de $G(z)$. Or on trouve

d'où résulte

$$
g_{1}(z)=\sum_{n=0}^{\infty} \ln (n+1) a_{n+1} \frac{\mathrm{I}}{(2 \pi i z)^{n+1}},
$$

$$
g_{1}(z)=2 \pi i z g(z)-a_{0} .
$$

Par conséquent nous avons

$$
F_{1}(z)=\int \frac{e^{2 \pi i z \zeta}-1}{e^{2 \pi i \zeta}-\mathrm{I}}\left(2 \pi i \zeta g(\zeta)-a_{0}\right) d \zeta
$$

puis, en comparant (34) et (36),

$$
F^{\prime}(z)=F_{1}(z)+a_{0} \int \frac{e^{2 \pi i z \zeta}-1}{e^{2 \pi i \zeta}-1} d \zeta+\int \frac{2 \pi i \zeta g(\zeta)}{e^{2 \pi i \zeta}-\mathrm{I}} d \zeta
$$

Ici la deuxième intégrale est une simple constante et la première s'ob-

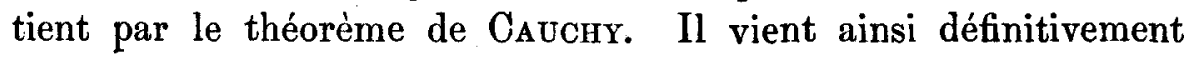

$$
F^{\prime}(z)=F_{1}(z)+a_{0} \sum_{k=-r}^{+r}\left(e^{2 \pi i k z}-1\right)+\int \frac{2 \pi i \zeta g(\zeta)}{e^{2 \pi i \zeta}-1} d \zeta
$$


Passons à une autre remarque. Soit $g(z)$ une fonction quelconque holomorphe pour $|z| \geq l$ et s'annulant pour $z=\infty$. Désignons par $r+\mathrm{I}$ le premier nombre entier positif supérieur à $l$ et formons la série

$$
\sum_{n=1+1}^{\infty}\left(g(n) e^{2 n \pi i z}+g(-n) e^{-2 n \pi i z}\right) . \quad \quad(n<z<1)
$$

Te dis que cette série est convergente et représente une fonction entière de $z$. En effet, soit pour $|z| \geq l$

$$
g(z)=\frac{c_{0}}{z}+\frac{c_{1}}{z^{2}}+\ldots+\frac{c_{n}}{z^{n+1}}+\ldots
$$

\section{Alors l'intégrale}

$$
G(z)=\int e^{2 \pi i z^{*}} g(\zeta) d \xi
$$

prise le long du cercle $|\zeta|=l$ représente une fonction entière de $z$, dont le développement suivant les puissances de $z$ sera

$$
G(z)=2 \pi i\left[c_{0}+r_{1}(2 \pi i z)+\ldots+\frac{r_{n}}{\left.\right|_{n} ^{n}}(2 \pi i z)^{n}+\ldots\right],
$$

puisque l'intégrale considérée se réduit au résidu de la fonction $e^{2 \pi i z r} g(\zeta)$ par rapport au point $\zeta=\infty$. Or en appliquant nos théorèmes à cette fonction $G(z)$ nous voyons que la série (38) est identique à une constante près au développement en série de Fouribr de la fonction entière

$$
-F(z)=-\int \frac{e^{2 \pi i z \xi}-\mathrm{I}}{e^{2 \pi i \xi}-\mathrm{I}} g(\zeta) d \zeta
$$

l'intégrale étant prise le long du cercle $|\zeta|=l$.

Maintenant je vais appliquer les théorèmes généraux à quelques exemples. Soit d'abord

$$
G(z)=2 \pi i e^{2 \pi i x z}=\sum_{n=0}^{\infty}(2 \pi i)^{n+1} \frac{x^{n}}{\mid \underline{n}} z^{n},
$$

où nous supposons pour éviter des discussions particulières que la con- 
Sur l'intégrale finie d'une fonction entière.

stante $x$, d'ailleurs arbitraire, ne soit pas un nombre entier. La série $g(z)$ devient

$$
g(z)=\sum_{n=0}^{\infty} \frac{x^{n}}{z^{n+1}}
$$

Elle est convergente et égale $\dot{\mathrm{a}} \frac{\mathrm{I}}{z-x}$ pour les valeurs de $z$ satisfaisant à la condition $|z|>|x|$.

Nous avons done

$$
F(z)=2 \pi i \sum_{n=0}^{\infty} \frac{(2 \pi i x)^{n}}{\mid \underline{n}}\left[\varphi_{n}(z)-\pi_{n, r}(z)\right]
$$

$v$ étant le plus grand nombre entier contenu dans $|x|$. De plus la fonction $F(z)$ est représentée par l'intégrale

$$
\int \frac{e^{2 \pi i z}-1}{e^{2 \pi i}-1} \frac{d \zeta}{\zeta-x}
$$

prise le long du cercle $|\zeta|=l$, où $l$ désigne une quantité positive comprise entre $|x|$ et $r+\mathrm{I}$. Le théorème de Cauchy nous donne l'expression explicite de $F(z)$, savoir

$$
F(z)=2 \pi i \frac{e^{2 \pi i x z}-\mathrm{I}}{e^{2 \pi i x}-\mathrm{I}}+\sum_{k=-r}^{k=+r}\left(e^{2 \pi i k z}-1\right) \frac{\mathrm{I}}{k-x} .
$$

En égalant les deux expressions (39) et (40) de $F(z)$, nous trouvons

(4 I) $\frac{e^{2 \pi i x z}-\mathrm{I}}{e^{2 \pi i x}-\mathrm{I}}=\frac{\mathrm{I}}{2 \pi i} \sum_{k=-r}^{k=+r}\left(\mathrm{I}-e^{2 \pi i k z}\right) \frac{\mathrm{I}}{k-x}+\sum_{n=0}^{\infty} \frac{(2 \pi i x)^{n}}{\underline{\underline{n}}}\left[\varphi_{n}(z)-\pi_{n, r}(z)\right]$,

équation valable pour toute valeur finie de $z$ et toute valeur de $x$ satisfaisant à l'inégalité $|x|<r+\mathrm{I}$.

Enfin, le développement en série de Fourier de $F(z)$ s'obtient d'après les formules (24) et (25)

$$
F(z)=\pi i+\sum_{k=r+1}^{\infty}\left\{\left(e^{2 \pi i k z}-\mathrm{I}\right) \frac{\mathrm{I}}{x-k}+\left(e^{-2 \pi i k z}-\mathrm{I}\right) \frac{\mathrm{I}}{x+k}\right\}
$$


et l'on trouve ainsi l'équation connue

$$
2 \pi i \frac{e^{2 \pi i z x}-\mathrm{I}}{e^{2 \pi i x}-\mathrm{I}}=\pi i+\sum_{k=1}^{\infty}\left|\left(e^{2 \pi i k z}-1\right) \frac{\mathrm{I}}{x-k}+\left(e^{-2 \pi i k z}-\mathrm{I}\right) \frac{\mathrm{I}}{x+k}\right| \cdot
$$

On en déduit aisément la suivante

$$
(0<z<1)
$$

$$
2 \pi i\left[\frac{e^{2 \pi i z x}-\mathrm{I}}{e^{2 \pi i x}-\mathrm{I}}-z\right]=\sum\left(e^{2-i k z}-\mathrm{I}\right)\left(\frac{\mathrm{I}}{x-k}+\frac{\mathrm{I}}{k}\right), \quad\left(\begin{array}{c}
k=-\infty+\ldots+\infty \\
k=0 \text { excepte }
\end{array}\right)
$$

dont nous ferons usage dans un instant, et qui présente l'avantage d'ètre applicable dans l'intervalle $0 \leqq z \leqq \mathrm{I}$. La série du second membre est en outre absolument convergente.

Considérons un deuxième exemple. Prenons cette fois

$$
G(z)=\frac{e^{2 \pi i z}-\mathrm{I}}{z}=\sum_{n=0}^{\infty} \frac{(2 \pi i)^{n+1}}{\underline{\underline{n}+\mathrm{I}}} z^{n}
$$

Nous trouvons

$$
g(z)=\sum_{n=0}^{\infty} \frac{\mathrm{I}}{n+\mathrm{I}} \frac{\mathrm{I}}{z^{n+1}}
$$

série convergente pour $|z|>$ I et qui a pour somme $\lg \frac{z}{z-\mathrm{I}}$.

Donc il vient

$$
F(z)=\sum_{n=0}^{\infty} \frac{(2 \pi i)^{n+1}}{\underline{n+1}}\left[\varphi_{n}(z)-\pi_{u, 1}(z)\right]=\int \frac{e^{2 \pi i z \zeta}-1}{e^{2 \pi i i_{5}}-1} \lg \frac{\zeta}{\zeta-1} d \zeta
$$

l'intégrale étant prise le long d'un cercle $|\zeta|=l$, dont le rayon $l$ est compris entre I et 2.

Le développement en série de Founier de $F(z)$ sera d'après (24)

$$
F(z)=\frac{\mathrm{I}}{2} \alpha_{0}-\sum_{n=2}^{\infty}\left|\lg \frac{n}{n-\mathrm{I}} e^{2-i n z}+\lg \frac{n}{n+\mathrm{I}} e^{-2 \pi i n z}\right|,
$$

ou la constante $\alpha_{0}$ se trouve au moyen de la formule (25)

$$
\left.\frac{\mathrm{I}}{2} \boldsymbol{\alpha}_{0}=\pi i+\sum_{n=2}^{\infty} \mid \lg \frac{n}{n-1}+\lg \frac{n}{n+\mathrm{I}}\right\}=\pi i+\lg 2 \text {. }
$$


Remarquons que la fonction

$$
\Psi(z)=\left(e^{2 \pi i z}-\mathrm{I}\right) \frac{\Gamma^{\prime}(z)}{\Gamma(z)}
$$

est une fonction entière satisfaisant à l'équation

$$
\Psi(z+\mathrm{I})-\Psi(z)=\frac{e^{2 \pi i z}-\mathrm{I}}{z} .
$$

Par conséquent la fonction $F(z)$ ne peut différer de $\Psi(z)$ que par une fonction entière $\varphi(z)$ ayant la période I. Maintenant je vais déterminer la fonction $\varphi(z)$ et réduire ainsi la fonction $F(z)$ à des fonctions élémentaires.

Dans ce but je reprends l'équation

$$
F(z)=\int \frac{e^{2 \pi i z_{5}^{5}}-\mathrm{I}}{e^{2 \pi i \zeta}-\mathrm{I}} \lg \frac{\zeta}{\zeta-1} d \zeta
$$

La détermination du logarithme qui figure au second membre doit étre choisie de façon que le logarithme s'annule pour $\zeta=\infty$. En excluant par une coupure rectiligne les valeurs réelles $0 \leqq \zeta \leqq \mathrm{I}$ nous pouvons prolonger analytiquement $\lg _{\frac{\zeta}{\zeta-1}}$ à l'intérieur de la courbe d'intégration. La différence des deux valeurs du logarithme aux deux bords de la coupure sera alors $2 \pi i$.

Cela posé, nous remarquons d'abord que la fonction sous le signe d'intégration a le pôle $\zeta=-$ I. En prenant le résidu par rapport à ce pôle nous pouvons écrire d'après le théorème de CAuchY

(47) $F(z)=\left(\mathrm{I}-e^{-2 \pi i z}\right) \lg 2+\int \frac{e^{2 \pi i z \zeta}-\mathrm{I}}{e^{2 \pi i \zeta}-\mathrm{I}} \lg \frac{\zeta}{\zeta-\mathrm{I}} d \zeta=\left(\mathrm{I}-e^{-2 \pi i z}\right) \lg 2+J$

l'intégrale $J$ s'étendant à un contour entourant la coupure $\zeta=0 \ldots$ I. Pour pouvoir réduire ce contour aux deux bords de la coupure, il faut transformer d'abord l'intégrale parce que la fonction qui multiplie le logarithme devient infinie pour $\zeta=\mathrm{I}$. C'est pourquoi nous écrivons

$$
J=\int\left[\frac{e^{2 \pi i z \zeta}-\mathbf{I}}{e^{2 \pi i \zeta}-\mathrm{I}}-\frac{e^{2 \pi i z}-\mathrm{I}}{2 \pi i(\zeta-\mathrm{I})}\right] \lg \frac{\zeta}{\zeta-\mathrm{I}} d \zeta
$$


Cette transformation est légitime puisque l'intégrale $\int \frac{1}{\zeta-1} \lg \frac{\zeta}{\zeta-1} d \zeta$ s'évanouit, la fonction à intégrer étant infiniment petite du deuxième ordre pour $\zeta=\infty$. Maintenant nous faisons tendre le chemin d'intégration vers les deux bords de la coupure $\zeta=0 \ldots$. Ainsi il vient

$$
J=\int_{0}^{1}\left(2 \pi i \frac{e^{2 \pi i x x}-1}{e^{2 \pi i x}-1}-\frac{e^{2 \pi i z}-\mathrm{I}}{x-\mathrm{I}}\right) d x
$$

ou encore

$$
\begin{gathered}
F(z)=\left(\mathrm{I}-e^{-2 \pi i z}\right) \lg 2+C\left(e^{2 \pi i z}-\mathrm{I}\right) \\
+2 \pi i \int_{0}^{1} \frac{d x}{e^{2 \pi i x}-1}\left[e^{2 \pi i z x}-\mathrm{I}-x\left(e^{2 \pi i z}-\mathrm{I}\right)\right],
\end{gathered}
$$

où nous avons posé

$$
\int_{0}^{1}\left[\frac{2 \pi i x}{e^{2 \pi i x}-1}-\frac{1}{x-1}\right] d x=C .
$$

Cela étant, nous décomposons $\frac{F(z)}{e^{2 \pi i z}-\mathrm{I}}$ en fractions simples en introduisant dans la formule

$$
\frac{F(z)}{e^{2 \pi i z}-1}=e^{-2 \pi i z} \lg 2+C+2 \pi i \int_{0}^{1} \frac{d x}{e^{2 \pi i x}-\mathrm{I}}\left[\frac{e^{2 \pi i z x}-\mathrm{I}}{e^{2 \pi i z}-\mathrm{I}}-x\right]
$$

le développement (comparez (43))

$$
2 \pi i\left[\frac{e^{2 \pi i z x}-\mathrm{I}}{e^{2 \pi i z}-\mathrm{I}}-x\right]=\sum\left(e^{2 k \pi i x}-\mathrm{I}\right)\left[\frac{\mathrm{I}}{z-k}+\frac{\mathrm{I}}{k}\right] .
$$

De cette manière nous trouvons

$$
\frac{F(z)}{e^{2 \pi i z}-\mathrm{I}}=e^{-2 \pi i z} \lg 2+C+\sum_{k=1}^{\infty}\left(\frac{\mathrm{I}}{z-k}+\frac{\mathrm{I}}{k}\right) .
$$


Sur l'intégrale finie d'une fonction entière.

Avant d'introduire ici la fonction $\Gamma(z)$, déterminons la constante $C$ en faisant $z=\frac{\mathrm{I}}{2}$ dans les équations (49) et (45). Il vient

$$
\begin{aligned}
-\frac{1}{2} F\left(\frac{\mathrm{I}}{2}\right) & =-\lg 2+C+\sum_{k=1}^{\infty}\left(\frac{-2}{2 k-\mathrm{I}}+\frac{2}{2 k}\right)=-3 \lg 2+C, \\
F\left(\frac{\mathrm{I}}{2}\right) & =\frac{\mathrm{I}}{2} \alpha_{0}-\sum_{n=2}^{\infty}(-\mathrm{I})^{n} \lg \frac{n \cdot n}{(n-\mathrm{I})(n+\mathrm{I})}=\pi i+\lg 2-\lg \left\{\frac{\mathrm{I}}{2}\left(\frac{\pi}{2}\right)^{2}\right\}
\end{aligned}
$$

où nous avons fait usage de la formule de WaLLIS. En réduisant nous obtenons

$$
C=\int_{0}^{\mathrm{I}}\left(\frac{2 \pi i x}{e^{2 \pi i x}-\mathrm{I}}-\frac{\mathrm{I}}{x-\mathrm{I}}\right) d x=\lg 2 \pi-\frac{\pi i}{2} .
$$

En remarquant maintenant que

$$
\sum_{k=1}^{\infty}\left(\frac{\mathrm{I}}{z-k}+\frac{\mathrm{I}}{k}\right)=\frac{\Gamma^{\prime}(\mathrm{I}-z)}{\Gamma^{\prime}(\mathrm{I}-z)}-\Gamma^{\prime}(\mathrm{I})=\frac{\Gamma(z)}{\Gamma(z)}-\Gamma^{\prime}(\mathrm{I})+\pi i \frac{e^{2 \pi i z}+\mathrm{I}}{e^{2 \pi i z}-\mathrm{I}},
$$

nous trouvons enfin

$$
F(z)=\left(e^{2 \pi i z}-1\right) \frac{\Gamma^{\prime}(z)}{\Gamma(z)}+\varphi(z)
$$

$\varphi(z)$ désignant la fonction entière périodique

(52) $\varphi(z)=\pi i+\frac{\pi i}{2}\left(e^{2 \pi i z}+\mathrm{I}\right)+\left(1-e^{-2 \pi i z}\right) \lg 2+\left(e^{2 \pi i z}-\mathrm{I}\right)\left(\lg 2 \pi-\Gamma^{\prime}(\mathrm{I})\right)$.

Les formules que nous venons d'écrire restent vraies si nous remplaçons l'unité imaginaire $i$ par $-i$. Par conséquent en séparant les parties réelles et imaginaires en considérant $z$ comme réel, nous trouvons des résultats restant valables pour toutes les valeurs réelles et complexes de $z$.

C'est ainsi que les équations (44), (51) et (52) nous donnent le théorème suivant:

Soit $\mathfrak{F}(z)$ la fonction entière définie par l'équation

$$
\mathscr{F}(z)=\sin \pi z \frac{\Gamma^{\prime}(z)}{\Gamma(z)}+\frac{\pi}{2} \cos \pi z+\left(\lg 2 \pi-\Gamma^{\prime}(\mathrm{I})\right) \sin \pi z .
$$


Nous aurons pour toute valeur finie réelle ou complexe de z les développements

$$
\left\{\begin{array}{c}
2 \sin \pi z \mathcal{F}(z) \\
=\lg 2 \cdot(\mathrm{I}-\cos 2 \pi z)+\sum_{n=0}^{\infty}\left[(-\mathrm{I})^{n} \frac{(2 \pi)^{2 n+2}}{\mid 2 n+2} \varphi_{2 n+1}(z)+\frac{\mathrm{I}-\cos 2 \pi z}{n+\mathrm{I}}\right] \\
2 \cos \pi z \mathcal{F}(z) \\
=-\pi+\lg 2 \cdot \sin 2 \pi z+\sum_{n=0}^{\infty}\left[(-\mathrm{I})^{n} \frac{(2 \pi)^{2 n+1}}{\mid 2 n+\mathrm{I}} \varphi_{2 n}(z)+\frac{2 \sin 2 \pi z}{2 n+1}\right] .
\end{array}\right.
$$

De l'équation (45) nous déduisons les développements en série de Fourier valables dans l'intervalle réel $\circ<z<\mathrm{I}$ :

$$
\left\{\begin{array}{l}
2 \sin \pi z \mathfrak{F}(z)=\lg \frac{1}{2} \cos 2 \pi z+\sum_{n=2}^{\infty} \lg \left(\frac{n^{2}}{n^{2}-1}\right) \cos 2 \pi n z \\
2 \cos \pi z \mathfrak{F}(z)=\lg \frac{1}{2} \sin 2 \pi z+\sum_{n=2}^{\infty} \lg \left(\frac{n-1}{n+1}\right) \sin 2 \pi n z
\end{array}\right.
$$

En ajoutant les équations (55) après les avoir multipliées respectivement par $\sin \pi z$ et $\cos \pi z$ on trouve aisément la belle série de M. Lerch: ${ }^{1}$

$$
\widetilde{F}(z)=\sum_{n=2}^{\infty} \lg \left(\frac{n}{n+1}\right) \sin (2 n+1) \pi z
$$

laquelle multipliée par $2 \sin \pi z$ et $2 \cos \pi z$ reproduit les équations (55). Remarquons encore que l'équation que nous avons trouvé plus haut, savoir

$$
2 \pi i \int_{0}^{1} \frac{d x}{e^{2 \pi i x}-\mathrm{I}}\left[\frac{e^{2 \pi i z x}-\mathrm{I}}{e^{2 \pi i 2}-\mathrm{I}}-x\right]=\sum_{k=1}^{\infty}\left(\frac{\mathrm{I}}{z-k}+\frac{\mathrm{I}}{k}\right),
$$

donne après quelques transformations faciles l'expression de la fonction entière $\sin \pi z \frac{d \lg \Gamma(z)}{d z}$ sous la forme d'une intégrale définie:

- Sur la différentiation d'une classe de séries trigonométriques, Annales scientifiques de l'Ecole Normale Supérieure, $3^{\text {me }}$ série, tome I2. (I 895.$)$ 


$$
2 \sin \pi z \frac{d \lg \Gamma(z)}{d z}
$$

$$
=2 \Gamma^{\prime}(\mathrm{I}) \sin \pi z-\frac{\sin \pi z}{z}-\pi \cos \pi z+\frac{1}{\pi} \int_{0}^{\pi}(x \sin \pi z-\pi \sin x z) \operatorname{tg} \frac{x}{2} d x .^{1}
$$

On vérifie d'ailleurs aisément cette formule en partant de la relation

$$
\frac{\sin x z}{\sin \pi z}-\frac{x}{\pi}=\frac{\mathrm{I}}{\pi} \sum_{k=1}^{\infty}(-\mathrm{I})^{k+1}\left(\frac{\mathrm{I}}{z+k}-\frac{\mathbf{I}}{z-k}-\frac{2}{k}\right) \sin k x
$$

laquelle multipliée par $\operatorname{tg} \frac{x}{2} d x$ et intégrée ensuite entre les limites o et $\pi$, donne le résultat

$$
\int_{0}^{\pi}\left(\frac{\sin x z}{\sin \pi z}-\frac{x}{\pi}\right) \operatorname{tg} \frac{x}{2} d x=\sum_{k=1}^{\infty}\left(\frac{1}{z+k}-\frac{\mathbf{I}}{k}\right)-\sum_{k=1}^{\infty}\left(\frac{\mathbf{I}}{z-k}-\frac{\mathbf{I}}{k}\right),
$$

qui ne diffère que par sa forme de l'équation (57).

Comme troisième exemple j'ai consideré l'équation

$$
F(z+1)-F(z)=\frac{\sin ^{2} \pi z}{z^{2}}
$$

qui est évidemment satisfaite pour

$$
F(z)=-\sin ^{2} \pi z^{2} \frac{d^{2} \lg I(z)}{d z^{2}}
$$

Mais les calculs étant un peu longs je me contente de transcire ici quelques formules que $\mathrm{j}$ 'ai obtenues et qui me paraissent dignes d'intérêt.

Ce sont les suivantes:

$$
\sin ^{2} \pi z \frac{d^{3} \lg \Gamma(z)}{d z^{2}}=\frac{\pi^{2}}{2}-\int_{0}^{\pi} \frac{\pi-x}{\sin x} \sin (2 z x-x) d x
$$

1. Cette formule (légèrement modifiée) a déjà été indiquée par MI. LERCH dans son mémoíre intitulé: Rüzné rýsledky $v$ theorii funkce gamma. (Rozpravy Ceské Akademie Cisare Frantiska Josefa, 28. Unora I896.) 
ou encore

$\left(59^{\prime}\right) \quad \sin ^{2} \pi z \frac{d^{2} \lg I(z)}{d z^{2}}=\frac{\pi^{2}}{2}+\frac{\sin ^{2} \pi z}{2 z^{2}}-\int_{0}^{\pi}(\pi-x) \cot x \cdot \sin 2 z x d x$.

Ces relations mettent en évidence que $\sin ^{2} \pi z \frac{d^{2} \lg \Gamma(z)}{d z^{2}}$ est une fonction entière; elles donnent de suite les séries entières de $2 z-\mathrm{I}$ et de $z$, représentant cette fonction dans tout le plan. Ainsi l'équation (59) donne

$$
\begin{gathered}
\sin ^{2} \pi z \frac{d^{2} \lg I(z)}{d z^{2}} \\
=\frac{\pi^{2}}{2}-c_{1}(2 z-1)+c_{2} \frac{(2 z-1)^{3}}{\mid \underline{3}}+\ldots \pm c_{n} \frac{(2 z-1)^{2 n-1}}{\mid 2 n-1} \mp \ldots,
\end{gathered}
$$

où nous avons posé pour abréger

$$
c_{n}=\int_{0}^{\pi} \frac{\pi-x}{\sin x} x^{2 n-1} d x .
$$

On déduit aussi très aisément de léquation (59) les coefficients de la série de Focrien représentant la fonction $\sin ^{2} \pi z \frac{d^{2} \lg \Gamma(z)}{d z^{2}}$ dans l'intervalle $o<z<\mathrm{I}$. Voici cette série

(61) $\sin ^{2} \pi z \frac{d^{2} \lg \Gamma(z)}{d z^{2}}=\frac{\pi^{2}}{2}+\pi \sum_{n=1}^{\infty}\left((n-\mathrm{I}) \lg \frac{n-\mathrm{I}}{n}+(n+\mathrm{I}) \lg \frac{n+\mathrm{I}}{n}\right) \sin 2 n \pi z$, ou il faut remplacer $(n-\mathrm{I}) \lg \frac{n-\mathrm{I}}{n}$ par zéro pour $n=\mathrm{I}$.

Revenons maintenant au cas général. L'équation

$$
F(z+1)-F(z)=G(z)
$$

est contenue comme cas particulier dans l'équation

$$
F(z+1)-a F(z)=G(z),
$$

a désignant une constante donnée, ou dans l'équation plus générale encore

$$
a_{0} F(z+n)+a_{1} F(z+n-1)+\ldots+a_{n-1} F(z+1)+a_{n} F(z)=G(z),
$$


$a_{0}, a_{1}, \ldots, a_{n}$ étant des constantes données. On peut réduire ces équations comme le fait M. Gurchakd, à l'équation $F(z+1)-F(z)=G(z)$. Mais on peut aussi les traiter directement d'une manière entièrement analogue à celle que nous avons employée plus haut pour l'équation $F(z+1)-F(z)=G(z)$.

Pour plus de simplicité considérons seulement l'équation (62)

$$
F(z+\mathrm{I})-a F(z)=G(z),
$$

où nous supposerons $a$ différent de $\mathrm{O}$ et de $\mathrm{I}$.

En premier lieu nous cherchons les fonctions rationnelles entières $\varphi_{n}(z)$ satisfaisant à l'équation

$$
\varphi_{n}(z+1)-a \varphi_{n}(z)=z^{n} .
$$

Remarquant que

$$
e^{\xi(z+1)}-a e^{\xi z}=e^{\xi z}\left(e^{\zeta}-a\right),
$$

nous voyons que ce sont les coefficients du développement de $\frac{e^{5 z}}{e^{\zeta}-a}$ suivant les puissances croissantes de $\zeta$, qui fournissent les fonctions $\varphi_{n}(z){ }^{1}$

Ainsi nous avons

$$
\frac{e^{r z}}{e^{\zeta}-a}=\sum_{n=0}^{\infty} \varphi_{n}(z) \frac{\zeta^{n}}{\mid \underline{n}}
$$

et par conséquent

$$
\varphi_{n}(z)=\frac{\mid n}{2 \pi i} \int \frac{e^{z z}}{e^{z}-a} \frac{d \zeta}{\zeta^{n+1}}
$$

l'intégrale étant prise autour du point $\zeta=0$ (le contour d'intégration ne contenant à son intérieur aucune des racines de l'équation $e^{\zeta}-a=0$ ). Maintenant considérons l'intégrale

$$
\psi_{n}(z)=\frac{n n}{2 \pi i} \int \frac{e^{\zeta z}}{e^{\tau}-a} \frac{d \zeta}{\zeta^{n}+1}
$$

prise le long d'un chemin entourant outre le point $\zeta=0$ un certain

1 Ce sont les fonctions considérées par M. Hermite daus ses intéressantes recherches sur les polynômes de Bernovilu. Voir: Sosis et Hermire, Sur les polynômes de Bernoulli, Journal de Crelle, tome II6, p. I33.

Acta mathematiea. 20. Imprimé le 2 férrier 1897. 
nombre de racines de l'équation $e^{5}-a=0$. L'équation (64) est encore satisfaite si nous remplaçons $\varphi_{n}(z)$ par $\phi_{n}(z)$. De plus $\phi_{n}(z)$ ne diffère de la fonction $\varphi_{n}(z)$ que par un certain nombre de termes de la forme

$$
-\frac{\mid n}{a} \frac{1}{(\lg a+2 k \pi i)^{n+1}} e^{(\lg a+2 k i i) z} \quad(k \text { entier }),
$$

qui sont les résidus provenant des racines de l'équation $e^{\zeta}-a=0$ qui se trouvent à l'intérieur du contour d'intégration. Ainsi nous avons

$$
\varphi_{n}(z)=\varphi_{n}(z)-\pi_{n}(z),
$$

$\pi_{n}(z)$ désignant une somme de fonctions exponentielles de la forme (68). Cela posé, je passe à la solution de l'équation

$$
F(z+1)-a F(z)=G(z),
$$

où je suppose que la fonction donnée

$$
G(z)=\sum_{n=0}^{\infty} a_{n} z^{n}
$$

est une fonction entière. On démontre, comme nous l'avons fait plus haut dans le cas $a=1$, qu'il est toujours possible de choisir les intćgrales $\psi_{n}(z)$ de façon que la série

$$
F(z)=\sum_{n=0}^{\infty} a_{n} \psi_{n}(z)=\sum_{n=0}^{\infty} a_{n}\left[\varphi_{n}(z)-\pi_{n}(z)\right]
$$

converge uniformément dans toute région finie du plan et représente par conséquent une fonction entière satisfaisant à l'équation (70).

Remarquons qu'en général il faut faire varier le chemin d'intégration de l'intégrale $\phi_{n}(z)$ avec l'indice $n$ et c'est ainsi que le nombre des termes exponentiels dont se compose $\pi_{n}(z)$ croit indéfiniment en même temps que l'indice $n$. L'unique cas où il suffit de prendre un chemin d'intégration fixe, c'est à dire indépendant de $n$, est celui où le rayon du cercle de convergence de la série $\sum_{n=0}^{\infty} \mid \underline{n} a_{n} z^{n}$ ne s'évanouit. pas.

Plaçons-nous dans ce cas et supposons que la série

$$
g(z)=\sum_{n=0}^{\infty} \mid \underline{n} a_{n} \frac{1}{z^{n+1}}
$$


soit convergente pour $|z|>l$. Désignons de plus par $C$ un cercle, dont le centre est ̀̀ l'origine, dont le rayon est supérieur à $l$ et qui n'a sur sa périphérie aucune racine de l'équation $e^{\zeta}-a=0$.

Alors nous pourrons prendre l'intégrale $\phi_{n}(z)$ le long du cercle $C$ et la fonction $F(z)$ se présentera sous la forme

$$
F(z)=\frac{\mathrm{I}}{2 \pi i} \int_{C} \frac{e^{\zeta z}}{e^{\zeta}-a} g(\zeta) d \zeta .
$$

Prenons pour exemple $a=-1$ et $G(z)=-\frac{\sin \pi z}{z}$, de sorte que l'équation à résoudre devient

$$
F(z+1)+F(z)=-\frac{\sin \pi z}{z}
$$

Elle est évidemment satisfaite par la fonction entière $\sin \pi z \frac{\Gamma^{\prime}(z)}{\Gamma(z)}$.

Désignons avec M. Hermite les fonctions $\varphi_{n}(z)$ correspondant à la constante $a=-\mathrm{I}$ par $\chi_{n}(z)$, de sorte que

$$
\frac{e^{\zeta z}}{e^{\zeta}+\mathrm{I}}=\sum_{n=0}^{\infty} \chi_{n}(z) \frac{\zeta^{n}}{\mid \underline{n}}
$$

La fonction $G(z)$ étant égale à

nous aurons

$$
-\frac{\sin \pi z}{z}=\sum_{n=0}^{\infty}(-1)^{n+1} \pi^{2 n+1} \frac{z^{2 n}}{2 n+1},
$$

$$
g(z)=\sum_{n=0}^{\infty}(-\mathrm{I})^{n+1} \frac{\pi^{2 n+1}}{2 n+1} \frac{\mathrm{I}}{z^{2 n+1}} \cdot
$$

Cette série est convergente pour $|z|>\pi$ et pour ces valeurs de $z$ sa somme est égale à $\frac{1}{2 i} \lg \frac{z-\pi i}{z+\pi i}$. Ainsi nous aurons d'après (74)

$$
F(z)=-\frac{\mathrm{I}}{4 \pi} \int \frac{e^{\zeta z}}{e^{\zeta}+\mathrm{I}} \lg \frac{\zeta+\pi i}{\zeta-\pi i} d \zeta
$$

l'intégrale étant prise le long du cercle $|\zeta|=l$, où $l$ est compris entre $\pi$ et $2 \pi$. 
En transformant cette intégrale d'une manière semblable à celle que nous avons employée plus haut pour l'intégrale (44), on reconnait que la fonction $F(z)$ est identique a la fonction $\mathscr{F}(z)$ définie par l'équation (53). Ainsi notre analyse nous donne comme solution de l'équation (75) la fonction

$$
F(z)=\sin \pi z \frac{\Gamma^{\prime \prime}(z)}{\Gamma^{\prime}(z)}+\frac{\pi}{2} \cos \pi z+\left(\lg 2 \pi-I^{\prime \prime}(\mathrm{I})\right) \sin \pi z .
$$

Le développement de cette fonction suivant les polynômes $\chi_{n}(z)$ sera d'après (72)

$$
F(z)=\sum_{n=0}^{\infty}(-1)^{n+1} \frac{\pi^{2 n+1}}{2 n+1} \psi_{2 n}(z)
$$

ou, puisque l'on a

$$
\begin{gathered}
\psi_{2 n}(z)=\frac{\mid 2 n}{2 \pi i} \int \frac{e^{-z}}{e^{j}+1} \frac{d \zeta}{\xi^{2 n+1}}=\chi_{2 n}(z)+2 \frac{2 n}{\pi^{2 n+1}}(-1)^{n+1} \sin \pi z, \\
F(z)=\sum_{n=0}^{\infty}\left[(-1)^{n+1} \frac{\pi^{2 n+1}}{2 n+1} \chi_{2 n}(z)+\frac{2 \sin \pi z}{2 n+1}\right] .
\end{gathered}
$$

Considérons encore l'équation

$$
F(z+\mathrm{I})-F(z)=\boldsymbol{\phi}(z)
$$

la fonction donnée $\phi(z)$ étant supposée méromorphe dans toute région finie du plan. Je vais démontrer que l'équation admet toujours une solution $F(z)$ elle-même méromorphe dans toute région finie du plan. Commençons par le cas spécial où il y a une droite verticale (c'est à dire parallèle à l'axe des quantités purement imaginaires) telle que tous les pôles de $\Phi(z)$ soient à gauche de cette droite. Je dis que l'on peut déterminer les fonctions entières rationnelles $g_{0}(z), g_{1}(z), \ldots$ de telle façon que la série

$$
\begin{gathered}
\mathrm{F}_{1}(z)=\left\{g_{0}(z)-\Phi(z)\right\}+\left\{g_{1}(z)-\phi(z+\mathrm{I})\right\}+\ldots \\
+\left\{g_{n}(z)-\phi(z+n)\right\}+\ldots
\end{gathered}
$$

représente une fonction méromorphe dans toute région finie du plan. 
En effet, les parties réelles des pôles de $\Phi(z)$ étant inférieures à un certain nombre $\rho$, celles des pôles de $\Phi(z+n)$ seront inférieures à $\rho-n$. Soit $k$ le plus petit nombre entier positif tel que $\rho-k$ est négatif; nous prendrons

$$
g_{0}(z)=g_{1}(z)=\ldots=g_{k-1}(z)=0 .
$$

Désignons maintenant par $\varepsilon_{k}, \varepsilon_{k+1}, \ldots$ des quantités positives assujetties à la seule condition que la série

$$
\varepsilon_{k}+\varepsilon_{k+1}+\varepsilon_{k+2}+\ldots
$$

soit convergente. La fonction $\boldsymbol{\phi}(z+n)$, où $n \geq k$, est holomorphe dans un cercle dont le centre est à l'origine et dont le rayon est certainement supérieur à $n-\rho$. Par conséquent nous aurons

$$
\Phi(z+n)=\mathfrak{P}_{n}(z),
$$

$\mathfrak{P}_{n}(z)$ désignant une série entière uniformément convergente pour les valeurs de $z$ satisfaisant à la condition $|z| \leqq n-\rho$. Cela posé, nous prendrons pour $g_{n}(z)$ un certain nombre de termes de la série $\mathfrak{P}_{n}(z)$ en disposant du nombre de ces termes de façon que l'inégalité

$$
\left|\Phi(z+n)-g_{n}(z)\right|<\varepsilon_{n}
$$

ait lieu pour toute valeur de $z$, dont le module est inférieur à $n-\rho$. Soit maintenant $R$ une région finie quelconque du plan. Comme la quantité $n-\rho$ croit indéfiniment avec $n$ il est évident qu'à partir d'un certain indice $n$, soit ̀̀ partir de $n=r$, l'inégalité $\left(8_{3}\right)$ eșt satisfaite pour toute valeur de $z$ appartenant à la région $R$. Donc la série

$$
\left\{g_{r}(z)-\Phi(z+r)\right\}+\left\{g_{r+1}(z)-\Phi(z+r+\mathrm{r})\right\}+\ldots
$$

est absolument et uniformément convergente dans la région $R$, d'où $\mathrm{il}$ suit que la série (82) représente une fonction méromorphe dans toute région finie du plan.

Maintenant la fonction $\mathrm{F}_{1}(z)$ satisfait évidemment à l'équation

$\mathrm{F}_{1}(z+\mathrm{I})-\mathrm{F}_{1}(z)=\Phi(z)-g_{0}(z)+\left\{g_{0}(z+\mathrm{I})-g_{1}(z)\right\}+\left\{g_{1}(z+\mathrm{I})-g_{2}(z)\right\}+\ldots$ c'est à dire à une équation de la forme

$$
\mathrm{F}_{1}(z+\mathrm{I})-\mathrm{F}_{1}(z)=\Phi(z)+G(z)
$$


$G(z)$ désignant une fonction entière. Soit $E(z)$ une fonction entière qui vérifie l'équation

$$
E(z+1)-E(z)=G(z)
$$

Alors la fonction

$$
F(z)=\mathrm{F}_{1}(z)-E(z)
$$

sera une solution méromorphe de l'équation (8I).

Remarquons encore que nous pouvons représenter $F(z)$ par une série de la même forme que $F_{1}(z)$, puisque $E(z)$ peut être développée (d'une infinité de manières) en une série toujours convergente procédant suivant des fonctions entières rationnelles. Ainsi dans le cas qui.nous occupe l'équation (8I) admet une solution méromorphe représentée par une série de la forme

$$
F(z)=\sum_{n=0}^{\infty}\left\{g_{n}(z)-\Phi(z+n)\right\}
$$

les $g_{n}(z)$ étant des fonctions entières rationnelles.

Considérons maintenant le cas où les pòles de la fonction donnée $\Phi(z)$ sont tous situés à droite d'une certaine droite verticale. Nous pouvons procéder d'une manière analogue et démontrer ainsi qu'il existe une solution méromorphe de l'équation (8I) représentée par une série de la forme

$$
F(z)=\sum_{n=1}^{\infty}\left\{\phi(z-n)-h_{n}(z)\right\}
$$

les $h_{n}(z)$ étant des fonctions entières rationnelles. D'ailleurs on ramène ce cas au cas précédent en remarquant que la fonction $-F(\mathrm{I}-z)$ sera une solution de l'équation $(81)$ si la fonction $F(z)$ vérifie l'équation $F(z+\mathrm{I})-F(z)=\Phi(-z)$.

Passons enfin au cas général. $\Phi(z)$ étant une fonction méromorphe donnée quelconque nous prenons à volonté une droite verticale et distribuons les pôles de $\Phi(z)$ en deux groupes, contenant l'un tous les pôles situés à droite, l'autre tous les pòles situés à gauche de la droite verticale. Les pôles situés sur cette droite peuvent ètre repartis à volonté dans l'un ou l'autre des groupes. D'après le théorème de M. Mrtrag-Leffren il est toujours possible de décomposer $\Phi(z)$ en deux fonctions $\Phi_{1}(z)$ et $\Phi_{2}(z)$

$$
\phi(z)=\Phi_{1}(z)+\Phi_{2}(z)
$$


de façon que les póles de $\Phi_{1}(z)$ et $\Phi_{2}(z)$ coïncident respectivement avec les pôles de $\Phi(z)$ appartenant au premier ou au deuxième groupe.

Maintenant déterminons les fonctions méromorphes

$$
F_{1}(z)=\sum_{n=1}^{\infty}\left\{\Phi_{1}(z-n)-h_{n}(z)\right\}, \quad F_{2}(z)=\sum_{n=0}^{\infty}\left\{g_{n}(z)-\Phi_{2}(z+n)\right\}
$$

telles que

$$
F_{1}(z+\mathrm{I})-F_{1}(z)=\Phi_{1}(z), \quad F_{2}(z+\mathrm{I})-F_{2}(z)=\Phi_{2}(z) .
$$

Alors la fonction

$$
F(z)=F_{1}(z)+F_{2}(z)
$$

satisfera à l'équation (8I). Ainsi notre théorème se trouve complètement démontré.

Remarquons encore que l'on peut construire d'une autre manière une solution méromorphe de l'équation (8I) dans le cas special où il existe une fonction entière $I I(z)$ ayant la période $\mathrm{I}$ et admettant tout póle de $\Phi(z)$ d'ordre $n$ comme zéro d'un ordre $\geqq n$. En effet, dans ce cas $\Pi(z) \Phi(z)$ sera une fonction entière et, en désignant par $\Psi(z)$ une fonction entière satisfaisant à l'équation $\Psi(z+1)-\mathscr{F}(z)=I(z) \Phi(z)$, la fonction méromorphe $F(z)=\frac{\Psi(z)}{\Pi(z)}$ sera une solution de l'équation $F(z+\mathrm{I})-F(z)=\phi(z)$.

Considérons maintenant le cas où tous les pôles de la fonction donnée $\Phi(z)$ sont du premier ordre, les résidus des póles étant en outre des nombres entiers positifs ou négatifs. Alors la fonction $F(z)$ définie par l'équation (89) sera une fonction méromorphe de mêrne espèce que $\Phi(z)$. Or toute fonction de cette espéce est la dérivée logarithmique d'une fonction méromorphe et réciproquement. Done l'équation $\frac{F^{\prime}(z+1)}{F^{\prime}(z+\mathrm{I})}-\frac{F^{\prime}(z)}{F^{\prime}(z)}=\frac{\Phi^{\prime}(z)}{\Phi(z)}, \Phi(z)$ désignant une fonction méromorphe donnée quelconque, admet une solution $F(z)$ elle-même méromorphe.

L'intégration nous conduit à la proposition suivante:

Si $\Phi(z)$ est 'une fonction méromorphe quelconque, il existe toujours une fonction méromorphe $F(z)$ telle que

$$
\frac{F(z+1)}{F^{\prime}(z)}=\Phi(z) .
$$


La solution la plus générale de cette équation sera évidemment $F(z) I(z)$, où $I I(z)$ désigne une fonction arbitraire admettant la période $\mathrm{I}$.

M. Guichard s'est occupé de la mème équation (90) en supposant que $\phi(z)$ est une fonction entière. Il a démontré que la condition nécessaire et suffisante pour qu'il existe une fonction entière $F(z)$ vérifiant l'équation (9o) consiste en ce que les zéros de $\Phi(z)$ soient tous situés à gauche d'une droite verticale (et non à )droite») comme cela est imprimé, par erreur sans doute, dans le mémoire de II. Gucharn).

Démontrons, pour finir, que l'équation

$$
\varphi_{1}(z) F(z+1)+\varphi_{0}(z) F(z)=\varphi(z)
$$

$\varphi(z), \varphi_{0}(z), \varphi_{1}(z)$ désignant des fonctions méromorphes données, admet toujours une solution $F(z)$ elle-mème méromorphe.

En eff't, d'après ce qui précede, nous pourrons déterminer la fonction méromorphe $F_{1}(z)$ telle que

$$
\frac{F_{1}^{\prime}(z+1)}{F_{1}(z)}=-\frac{\varphi_{1}(z)}{\varphi_{0}(z)}
$$

Alors l'équation à résoudre peut s'écrire ainsi:

$$
F_{1}(z+\mathrm{I}) F(z+\mathrm{I})-F_{1}(z) F(z)=-\frac{\varphi(z) F_{1}(z)}{\varphi_{0}(z)} .
$$

Donc en désignant par $F_{2}(z)$ une fonction méromorphe vérifiant l'équation

$$
F_{2}(z+1)-F_{2}(z)=-\frac{\varphi(z) F_{1}^{\prime}(z)}{\varphi_{0}(z)},
$$

il est évident que la fonction

$$
F(z)=\frac{F_{2}(z)}{F_{1}^{\prime}(z)}
$$

sera une solution méromorphe de l'équation ( $9 \mathrm{I}$ ).

Zürich, I 4 juillet I 896 .

Je profite de cette occasion pour ajouter à mon mémoire : ̈̈ber die Anzahl der Classen bịärer quadratischer Formen von negativer Determinante» (Tome 19 de ce journal) une citation que je dois ¿ l'obligeance de II. Fronexrus. I'idée de déterminer les sommes de signes de Legexpre par les résidus des coefficients dans le développement de fonctions élémentaires se trouve déjà dans le grand mémoire de Cacchy „Sur la théolie des nombres». (Mémoires de l'A cadémie royale des sciences de l'Institut de France, 1810, tome 17.) 